\title{
EFFECTS OF DIFFERENT FLAME RETARDANT TREATMENTS ON THE COMBUSTIBILITY OF BAMBOO FILAMENT
}

\author{
Chunyan Li, Xinjie Zhou, Changhao Yang, Lili Yu \\ Tianjin University Of Science And Technology \\ China \\ Hui Li \\ ${ }^{1}$ Hubei Academy Of Forestry \\ 2National Positioning Observation And Research Station Of Bamboo Forest \\ Ecosystem In Mufu Mountain \\ China \\ Benhua Fei \\ International Center For Bamboo And Rattan \\ China \\ (RECEIVED MAY 2020)
}

\begin{abstract}
Bamboo filaments were treated with boric acid and borax (the mass ratio of 1:1, the concentration of 20\%) with four different treatment methods including atmospheric immersion, cold and hot bath immersion, vacuum impregnation and vacuum-pressure impregnation. The different treatment methods on the boron loading were analyzed and the corresponding flame resistance of bamboo filaments were evaluated by the cone analysis. The results showed that suitable treatment method with optimized processing indexes, such as hot and cold bath immersion in the condition of $100^{\circ} \mathrm{C} / 2 \mathrm{~h}$ and $20^{\circ} \mathrm{C} / 2 \mathrm{~h}$ with 3 cycles, was more credible to accelerate the percentages of boron loading in the bamboo filaments, and the lowest result was found in the samples with vacuum impregnation. Compared to the untreated samples, the heat and smoke release would be decreased significantly, especially for the samples with the promising hot and cold treatment, and promising pressure treatment, attributed to the more stable boron fixed in the bamboo filaments.
\end{abstract}

KEYWORDS: Bamboo, flame resistance, boron loading, flame-retardant treatment, combustibility.

\section{INTRODUCTION}

As one of the most important substitutes for wood, bamboo has been widely used in the fields of decoration, furniture and building materials due to its excellent mechanical properties, short growth cycle, strong regeneration ability, longer fiber length, good elasticity and superior carbon fixation ability (Verma et al. 2012, Subekti et al. 2018, Zhu et al. 2019). 
However, as one of the flammable substances, the combustibility of bamboo has become the major obstacle for its utilization (Du et al. 2014, Zhou et al. 2018), for examples, once the bamboo was ignited, the flame and toxic gases would fill in the whole room within 5 to 10 minutes (Zheng et al. 2019). As the result, it is critical to perform suitable flame-retardant treatment to guarantee the application safety of bamboo-based materials (Zheng et al. 2016, $\mathrm{Li}$ et al. 2018, Fang et al. 2020). However, the permeability of bamboo is very poor, which is attributed to the vertical penetration mainly depending on the tube and screen (Kučerová 2012, Yu et al. 2016), as the result, it is difficult to be treated with the flame retardants to reach the suitable retention (Yong et al. 2013, Wen 2017). Many researches involving in the sample pre-treatments and the flame-retardant treatment methods have been performed to improve the retention in bamboo. The pre-treatments such as reducing the thickness of the samples, selecting the suitable indexes of the flame retardants including the active ingredients, $\mathrm{pH}$, concentration, have obtained some promising results in some researches (Shu 2010, Li et al. 2011). Additionally, some other researchers have paid more attentions to modify the permeability of bamboo by many physical and chemical measures, such as microwave treatment, pressure treatment, freeze-drying treatment, extraction, etc. (Guan et al. 2013, Haase et al. 2018). $\mathrm{Xu}$ et al. (2018) found that vacuum freeze-drying increased the porosity of bamboo to $73 \%$, and it had no obvious effect on the mechanical properties of bamboo. The ultrasonic treatment of bleached and carbonized bamboo increased the surface free energy and improved the wettability and permeability of bamboo (Huang 2017). In addition, hydrochloric acid (2\%) hydrolysis and ethanol extraction could also effectively improve the permeability of bamboo (Rao et al. 2013).

The direct and effective means to increase the flame-retardant retention are to perform suitable flame-retardant treatment methods with appropriate processing indexes. Recently, the widely used flame retardant treatment methods of bamboo in the industry could be summarized as immersion and surface coating. The most commonly used immersion methods include atmospheric immersion, hot and cold bath immersion and vacuum/and pressure impregnation (Du et al. 2016, Yu et al. 2017). Surface coating is another convenient method for bamboo flame retardant treatment. The flame retardant or flame-retardant coating is directly sprayed or painted on the surface of bamboo materials or bamboo products to form a thinner film, which can effectively obstruct the heat and oxygen from bamboo. However, the coating film was so thin that the active flame-retardant gradients were limited, and it was vulnerable to be damaged by mechanical force (Jin et al. 2015, Li et al. 2018).

In order to clarify the difference in the flame resistance of bamboo performed with different treatment methods, four frequently used flame retardant treatment methods including atmospheric immersion, cold and hot bath immersion, vacuum impregnation and vacuum-pressure immersion were performed in this research, and based on the results, it was conducted to determine the correlation between boron loading and flame resistance with different treatment conditions as well as to obtain the suitable processing indexes with the promising treatment method.

\section{MATERIAL AND METHODS}

\section{Samples}

Moso bamboo (Phyllostachys edulis (Carr.) H.de Lehaie) was taken from Hubei Province, China. After air-drying, the moso bamboo was cut along the direction of the fiber to obtain the bamboo splits, then to cut into the bamboo filaments. The filaments were stuck together with non-woven fabric by a polyvinyl acetate adhesive and cut into small pieces with dimensions of 
$100 \mathrm{~mm}$ (tangential) $\times 100 \mathrm{~mm}$ (longitudinal) $\times 1.5 \mathrm{~mm}$ (radial). Bamboo filaments with similar weights were selected as the test samples. The mass fraction of boric acid and borax fire retardant was $20 \%$, and the ratio between boric acid and borax was 1:1.

\section{Flame retardant treatments}

Bamboo filaments were dried to constant weight in a drying oven at $60^{\circ} \mathrm{C}$. Then bamboo filaments were taken out and cooled to weight and recorded. Bamboo filaments were treated with four different flame-retardant treatments including atmospheric immersion, cold and hot bath immersion, vacuum impregnation and vacuum-pressure impregnation with different processing indexes as showed in Tab. 1, and six repeated specimens were set in each treatment. In the atmospheric immersion treatments, the bamboo filaments were immersed into the flame retardant at 20,60 , and $100^{\circ} \mathrm{C}$, respectively. In the cold and hot bath immersion treatments, the bamboo filaments were first immersed in hot flame retardant at $100^{\circ} \mathrm{C}$ for $0.5,1$, and $2 \mathrm{~h}$, respectively, and then replaced them into the cold flame retardant for $0.5,1$, and $2 \mathrm{~h}$, respectively. In the vacuum impregnation, the bamboo filaments were put into a beaker and the flame retardant was introduced into the beaker to immerse the samples at room temperature by the vacuum pressure $(-0.1 \mathrm{MPa})$. In the vacuum-pressure impregnation, the bamboo filaments were put in a closed vacuum pressurized tank, which was vacuumed to $-0.1 \mathrm{MPa}$ for $30 \mathrm{~min}$, and introduced the flame retardant to the tank, then pressurized to $0.8 \mathrm{MPa}$ for $1 \mathrm{~h}$. After different flame-retardant treatments, then bamboo filaments were dried to constant weight in a drying oven at $60^{\circ} \mathrm{C}$. The specimens would be treated with the same recycle for 1 to 4 cycles.

Tab. 1: Fire retardant immersion conditions for bamboo filament.

\begin{tabular}{|c|c|c|c|c|c|}
\hline Treatment method & $\begin{array}{c}\text { Temperature } \\
\left({ }^{\circ} \mathrm{C}\right)\end{array}$ & $\begin{array}{c}\text { Treatment } \\
\text { duration (h) }\end{array}$ & $\begin{array}{c}\text { Vacuum } \\
\text { (MPa) }\end{array}$ & $\begin{array}{c}\text { Pressure } \\
\text { (MPa) }\end{array}$ & $\begin{array}{c}\text { Treatment } \\
\text { cycles }\end{array}$ \\
\hline Atmospheric immersion & $20,60,100$ & 2 & & & \multirow{4}{*}{$1,2,3,4$} \\
\hline Cold and hot bath immersion & 20,100 & $0.5,1,2$ & & & \\
\hline Vacuum impregnation & 20 & 1 & -0.1 & & \\
\hline Vacuum-pressure impregnation & 20 & 1 & -0.1 & 0.8 & \\
\hline
\end{tabular}

Percentages of boron loading (BL) in the samples were calculated using Eq. 1:

$B L=\frac{W_{2}-W_{1}}{W_{1}} \times 100 \%$

where: W1 and W2 are the weights of each specimen before and after the flame-retardant treatment.

\section{Combustibility by cone calorimeter}

After different flame-retardant treatments, the bamboo filaments were dried to constant weight in a drying oven at $60^{\circ} \mathrm{C}$. The combustibility of the samples with the highest boron loading in each flame-retardant treatment method were selected and evaluated by a cone calorimeter according to ISO 5660 (2002). Six specimens with the same treatment condition were prepared with dimensions of $100 \times 100 \times 1.5 \mathrm{~mm}$, and placed horizontally under a cone heater with a heat flux of $50 \mathrm{~kW} \cdot \mathrm{m}^{-2}$. A stainless-steel cover with an opening of $0.0088 \mathrm{~m}^{2}$ on the upper part was attached. The data was recorded by a computer on a second basis. 


\section{RESULTS AND DISCUSSION}

\section{Effects of different treatment methods on boron loading}

Atmospheric immersion

As showed in Fig. 1, the immersion temperature played a significant effect on the percentages of boron loading in the bamboo filament. At ambient temperature (about $20^{\circ} \mathrm{C}$ ), the percentages of boron loading were less than $8 \%$, however, when the temperature was up to $100^{\circ} \mathrm{C}$, it could reach more than $14 \%$ in all the samples with different treatment cycles.

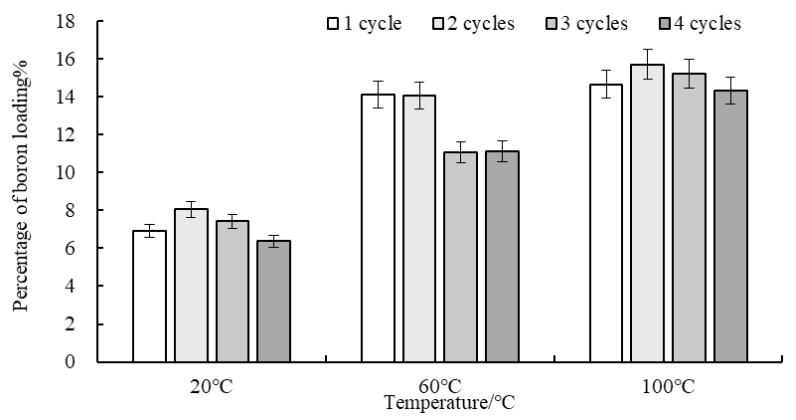

Fig. 1: Effect of different atmospheric immersion treatments on the boron loading of bamboo filaments.

Additionally, the treatment cycles of the flame-retardant treatments also had a certain effect on the boron loading. However, it was not the more, the better, and the maximum boron loading was observed in the samples with 2 cycles of immersion treatments, which was attributed to that too many treatments would increase the leaching risk of the flame retardants during treatment. In the atmospheric immersion, the maximum percentage of boron loading was observed in the samples treated at $100^{\circ} \mathrm{C}$ for $2 \mathrm{~h}$ with 2 cycles of treatments, and it could reach $15.72 \%$.

\section{Hot and cold bath immersion}

As showed in Fig. 2, hot and cold bath immersion was more favorable to increase the boron loading of bamboo filaments than atmospheric immersion, and the percentages of boron loading of all the samples were above $14 \%$. In the hot and cold bath immersion, the processing duration and the cycles of cold and hot bath immersion played significant effects on the boron loading of bamboo filaments. The similar results could be observed that the increase of treatment cycles was not always conducive to improve the boron loading of bamboo filament, and the boron loading in all the samples with four cycles of treatments began to decrease. In cold and hot bath treatment, the better results were observed in the samples treated with twice or three cycles of treatments. The maximum percentages of boron loading in the same treatment condition with different treatment cycles were showed in Tab. 2. It could be seen that the duration of hot bath immersion was not as obvious as cold bath immersion on the boron loading of bamboo filaments, and the percentages of boron loading changed slightly as the hot bath immersion increased in the same cold bath immersion. The results indicated that the extension of cold bath immersion was the key factor to increase the boron loading of bamboo filaments in the cold and hot bath immersion, because during the hot bath immersion, the thinner bamboo filaments could remove the internal air in a very short time to achieve the vacuum state, as the result, the samples could quickly become saturated with the flame retardant. The boron loading of bamboo filament increased obviously with the extension of the cold bath immersion, which was due to the slow absorption rate of the cold flame-retardant immersion. 


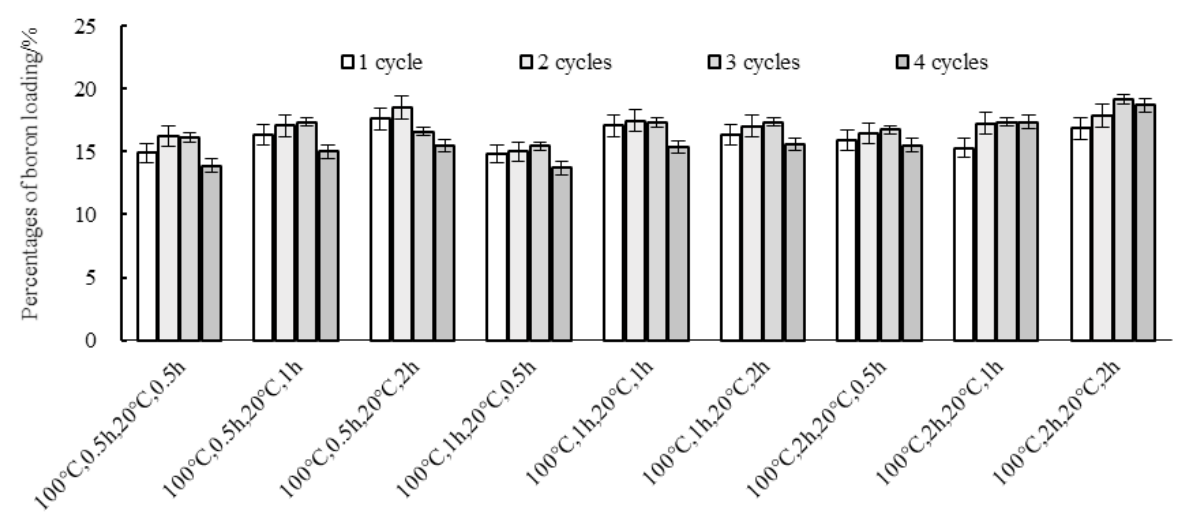

Fig. 2: Effect of different cold and hot bath immersion treatments on the boron loading of bamboo filaments.

The maximum percentages of boron loading in the same treatment condition with different treatment cycles were showed in Tab. 2. It could be seen that the duration of hot bath immersion was not as obvious as cold bath immersion on the boron loading of bamboo filaments, and the percentages of boron loading changed slightly as the hot bath immersion increased in the same cold bath immersion. The results indicated that the extension of cold bath immersion was the key factor to increase the boron loading of bamboo filaments in the cold and hot bath immersion, because during the hot bath immersion, the thinner bamboo filaments could remove the internal air in a very short time to achieve the vacuum state, as the result, the samples could quickly become saturated with the flame retardant. The boron loading of bamboo filament increased obviously with the extension of the cold bath immersion, which was due to the slow absorption rate of the cold flame-retardant immersion.

Tab. 2: Effects of different cold and hot bath immersion treatments on the maximum boron loading of the bamboo filaments.

\begin{tabular}{ccccc}
\hline \multirow{2}{*}{ Treatment conditions } & \multicolumn{4}{c}{$\mathbf{2 0}^{\circ} \mathbf{C}$} \\
\cline { 2 - 5 } & & $\mathbf{0 . 5} \mathbf{h}$ & $\mathbf{1 ~ h}$ & $\mathbf{2 ~ h}$ \\
\hline \multirow{2}{*}{$100^{\circ} \mathrm{C}$} & $0.5 \mathrm{~h}$ & $16.21(0.95)$ & $17.35(0.72)$ & $18.55(1.29)$ \\
& $1 \mathrm{~h}$ & $15.45(1.02)$ & $17.45(1.99)$ & $17.36(1.27)$ \\
& $2 \mathrm{~h}$ & $16.73(1.53)$ & $17.37(1.27)$ & $19.16(0.78)$ \\
\hline
\end{tabular}

\section{Vacuum impregnation}

Percentages of boron loading in the bamboo filaments with vacuum impregnation were showed in Fig. 3. It can be seen that the treatment cycles played an obvious role in the vacuum impregnation. The percentage of boron loading of bamboo filaments with two cycles were the highest and it could reach $10.54 \%$, which was significantly lower than those with cold and hot bath immersions. The reason was mainly caused by the lower negative pressure generated by the vacuum. 


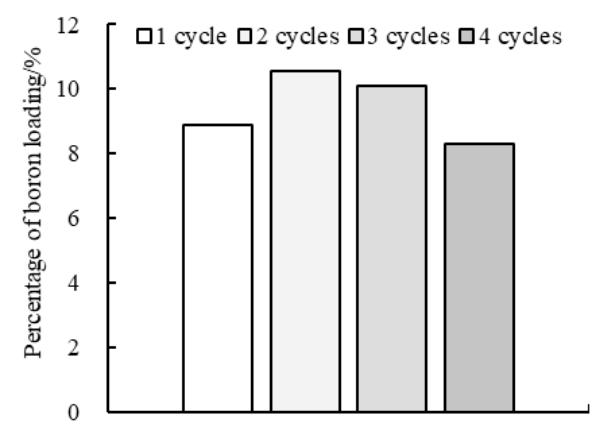

Fig. 3: Effect of different vacuum impregnation cycles on the boron loading of bamboo filaments.

\section{Pressure impregnation}

Due to the limitation of pressure equipment, only the effect of different flame-retardant impregnation cycles on the boron loading of bamboo filaments were investigated in this study. As shown in Fig. 4, the percentages of boron loading were increased insignificantly with the flameretardant cycles increased, and it could be up to $15.52 \%$ in the samples with 4 cycles of pressure impregnation. The result demonstrated that the flame retardant could have more opportunities to penetrated into bamboo filaments with the favor of higher pressure.

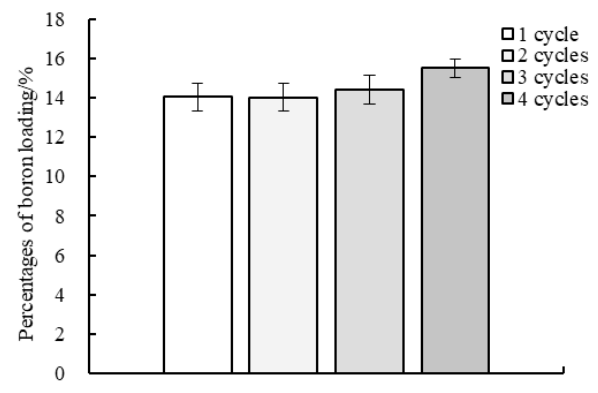

Fig. 4: Effect of different pressure impregnation cycles on the boron loading of bamboo filaments.

\section{Combustibility analysis}

Heat release analysis

The heat release rates (HRR) and the total amounts of heat release (THR) for the bamboo filaments with different flame-retardant treatments are shown in Figs. 5a,b.

Compared with untreated samples, different flame-retardant treatments could effectively reduce the HRR during the whole combustion process, which indicated that boric acid/borax could effectively block heat and oxygen by the thin layer of melting primevally to reduce the rate of heat release during combustion process, which was similar with other research that boron based complexes had excellent flame retardant performance ( $\mathrm{Li}$ et al. 2018). However, the difference in the samples treated with these four flame retardant treatment methods respectively were very obvious. At $0-35 \mathrm{~s}$, the HRR of bamboo filaments treated by cold and hot bath were the lowest among all the samples, while at 35-45 s, the HRR of bamboo filaments treated by pressure decreased sharply. The results that the samples with higher boron loading 
or/and suitable treatment were both important to reduce the heat release during combustion process, which was also proved by the results observed in the whole process of combustion that the samples with the vacuum treatment had the worst flame retardance, while the HRR in the samples with hot and cold bath immersion and pressure impregnation were decreased significantly.

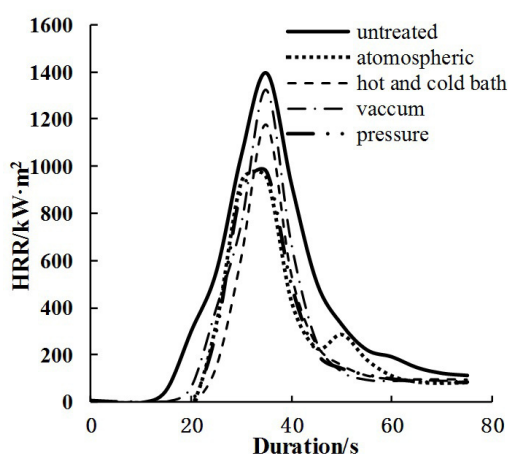

(a) Heat release rate.

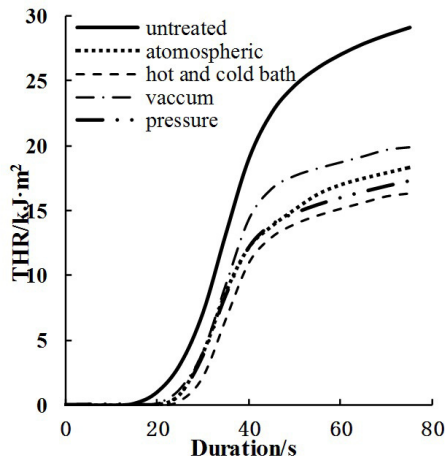

(b) Total heat release.

Fig. 5: HRR curves of bamboo filaments with different treatments.

As observed from Fig. 5b, the process of bamboo filament combustion can be divided into three stages according to the change of heat release: early stage of combustion $(0-25 \mathrm{~s})$, middle stage of combustion (25-45 s) and final stage of combustion (45-75 s). In the early stage, the THR of all the treated samples were lower than those in the untreated samples, but the difference in THR among different flame-retardant treatments could be negligible. In the middle stage, the flame-retardant treatments had a significant different effect on the THR of bamboo filaments, which seemed that the best flame resistance were observed in the samples with hot and cold immersion, followed by pressure impregnation. At the final stage, the THR of all the bamboo filaments increased gently. From the whole combustion process, the bamboo filament treated by immersion, cold and hot bath, vacuum and pressure flame retardant decreased by $36.94 \%, 44.01 \%, 31.67 \%$ and $40.65 \%$, respectively, which also demonstrated that cold and hot bath immersion and pressure impregnation were suitable treatment methods for bamboo filaments.

\section{Smoke release analysis}

The total smoke release (TSP) of bamboo filaments treated with different flame-retardant treatments was showed in Fig. 6. It can be seen that the TSP in all the treated bamboo filaments was significantly reduced. Compared to the untreated samples, TSP in the bamboo filaments treated with immersion, cold and hot bath, vacuum and pressure flame retardant could decreased by $91.30 \%, 89.49 \%, 83.93 \%$ and $89.67 \%$, respectively. Except for the slightly higher smoke emission of vacuum treated bamboo filaments, the other flame-retardant treated bamboo filaments had excellent smoke inhibition, while the difference among different treatment methods were very slight. This result further proved that the reasonable flame retardant was essential to reduce the smoke emission caused by the combustion of bamboo. 


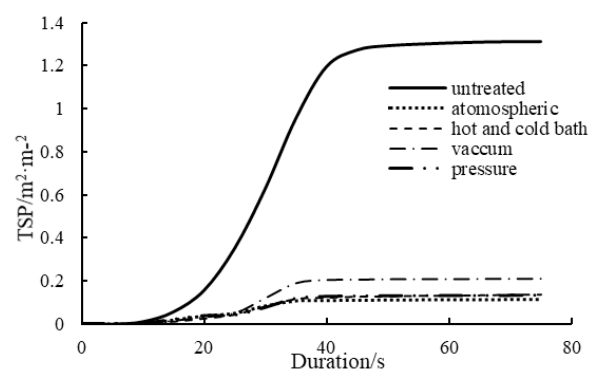

Fig. 6: TSP-time curves of bamboo filament with different treatments.

The average amount of smoke release from per mass (ASEA) and the toxic gases of $\mathrm{CO}$ and $\mathrm{CO}_{2}$ yield in the bamboo filaments during the combustion process were showed in Tab. 3. As observed, compared to the untreated samples, different flame-retardant treatments could significantly reduce the ASEA value of all the treated samples. Bamboo filaments treated by immersion, cold and hot bath, vacuum and pressure flame retardant would decrease by $86.90 \%, 73.66 \%, 65.68 \%$ and $77.21 \%$, respectively. The results indicated different flame retardants would change the thermal decomposition process of bamboo filaments and affect the visible smoke production of bamboo filaments, and just as other researchers showed the smoke release were changed to some flame-retardant gases such as $\mathrm{NH}_{3}$ and $\mathrm{CO}_{2}$ (Wang et al. 2004, Zhang et al. 2018).

Tab. 3: Smoke production parameters with different flame-retardant treatments.

\begin{tabular}{lccccc}
\hline \multicolumn{1}{c}{ Smoke emission parameter } & Untreated & $\begin{array}{c}\text { Atmospheric } \\
\text { immersion }\end{array}$ & $\begin{array}{c}\text { Cold and hot } \\
\text { bath }\end{array}$ & $\begin{array}{c}\text { Vacuum } \\
\text { impregnation }\end{array}$ & $\begin{array}{c}\text { Pressure } \\
\text { immersion }\end{array}$ \\
\hline ASEA $/\left(\mathrm{m}^{2} \bullet \mathrm{kg}^{-1}\right)$ & 85.84 & 11.25 & 22.61 & 29.46 & 19.57 \\
Average $\mathrm{CO}$ yield / $\left(\mathrm{kg} \bullet \mathrm{kg}^{-1}\right)$ & 0.1455 & 0.0527 & 0.0715 & 0.0667 & 0.0669 \\
Average $\mathrm{CO}_{2}$ yield / $\left(\mathrm{kg} \bullet \mathrm{kg}^{-1}\right)$ & 3.6101 & 2.0914 & 1.9516 & 1.6827 & 2.2654 \\
\hline
\end{tabular}

As Tab. 3 showed, compared to the untreated samples, bamboo filaments treated with different flame-retardant treatments had significantly reduced the $\mathrm{CO}$ and $\mathrm{CO}_{2}$ production rates, which was mainly due to the fact that during the dehydration reaction, the volatile products during the combustion contained less $\mathrm{CO}_{2}$ and more water. It seemed that different flameretardant treatment methods had slight effect on the $\mathrm{CO}$ and $\mathrm{CO}_{2}$ yield compared with the value of ASEA.

\section{CONCLUSIONS}

Because of the poor permeability of bamboo, it is essential to perform some modifications to improve the flame retardant of bamboo. Different flame-retardant treatments including atmospheric immersion, cold and hot bath immersion, vacuum impregnation and vacuumpressure impregnation had obvious difference in the boron loading of bamboo filaments attributed to the permeability modification difference. Suitable treatment method with optimized processing indexes including treatment duration, temperature and cycles was more 
propitious to increase the percentages of boron loading in the bamboo filaments, which was also demonstrated the reasonable conditions for improving the permeability of bamboo. Compared to the untreated samples, the indexes of heat and smoke were reduced dramatically in all the boron-treated bamboo filaments during the combustion process, and would be affected by the flame treatment methods. These improvements were attributed to the permeability of the flame retardants into bamboo. Generally, the samples with the vacuum treatment had the worst heat suppressibility, while the samples with hot and cold bath immersion and pressure impregnation had better performance, which was also attributed to the significant permeability difference of flame retardants into bamboo. The similar results were also observed in the ASEA values, however the effects of different treatment methods on the $\mathrm{CO}$ and $\mathrm{CO}_{2}$ yield were not obvious.

\section{ACKNOWLEDGMENT}

The authors are grateful for the financial support of the Subjects of National Natural Science Foundation of China (NSFC No. 31400499), the National College Students Innovation and entrepreneurship training program (201910057047), and the Science and Technology of Tianjin University College Students Innovation and entrepreneurship training program (202110057013).

\section{REFERENCES}

1. Verma, C.S., Chariar, V.M., 2012: Development of layered laminate bamboo composite and their mechanical properties. Composites Part B: Engineering 43(3): 1063-1069.

2. Subekti, N., Widiyaningrum, P., Yoshimura, T., Fibriana, F., 2018: The strength and termite resistance characteristics of fiberboards produced from the renewable bamboo biomass. Wood Research 63(3): 409-418.

3. Zhu, Z.Z., Zhu, S.L., Zhang, T.T., Guo, Y., Liu, S.Q. Liu, H.R., Chen, Y.X., 2019: A brief analysis on the design of detachable furniture made of bamboo oriented strand board (BOSB). Journal of Bamboo Research 38(2): 70-74.

4. Du, C.G., Song, J.G., Chen, Y.X., 2014: The effect of applying methods of fire retardant on physical and mechanical properties of bamboo scrimber. Advanced Materials Research 1048: 465-468.

5. Zhou, Z.X., Yao, X.L., Du, C.H., Yu, H.L., Huang, Q.L., Liu, H.Z., 2018: Effect of hygroscopicity of fire retardant on hygroscopicity of fire retardant bamboo chips. Wood Research 63(3): 373-382.

6. Zheng, M.H., Wang, K., Wu, Q.Zhang, W.B.,Zhang, X.C.,Li, C.B.,Zhou, Z.C., Zhang, S.H., 2016: The performances of bamboo treated with three flame retardants. Journal of Bamboo Research 35(4): 8-13.

7. Fang, L., Lu, X.Z., Zeng, J., Chen, Y.Y., Tang, Q.H., 2020: Investigation of the flame-retardant and mechanical properties of bamboo fiber-reinforced polypropylene composites with melamine pyrophosphate and aluminum hypophosphite addition. Materials 13(2): 479-491.

8. Li, H., Chen, M.L., Lv H.F., Yang, F., Yu, L.L., Fei, B.H., Ma, X.X., 2018: Effects of guanylurea phosphate treatment on the performance of decorative bamboo filament. Bioresources 13(2): 3487-3499.

9. Kučerová, I., 2012: Methods to measure the penetration of consolidant solutions into 'dry' wood. Journal of Cultural Heritage 13(3): 191-195. 
10. Wen, X.W., 2017: Study on preservative penetration of bamboo and its influencing factors. Master's thesis. Pp 25-32, Chinese Academy of Forestry, Beijing, China.

11. Yong, C., Zhou, M.M., Guan, M.J., 2013: Effect of ultrasonic cavitation on microscopic structure and starch granule of bamboo. China Forestry Science and Technology 27(3): 99-102.

12. Li, Y.F., Liu, Y.X., Wang, F.H., Gang, G.J., 2011: Controlling factors of wood permeability and its improving measures. Scientia Silvae Sinicae 47(5):131-139.

13. Shu, T.T., 2010: The research of moso-bamboo dye solution permeability. Master's thesis. Pp 31-35, Zhejiang A\&F University, Zhejiang, China.

14. Haase, J., Leung, L., Evans, P., 2018: Plasma pre-treatments to improve the weather resistance of polyurethane coatings on black spruce wood. Coatings 9(8): 1-14.

15. Guan, M.J., Yong, C., Wang, L., 2013: Shear strain and microscopic characterization of a bamboo bonding interface with poly(vinyl alcohol) modified phenol-formaldehyde resin. Journal of Applied Polymer Science 130(2): 1345-1350.

16. Xu, J., He, S., Li, J., Yu, H., Zhao, S., Chen, Y., Ma, L., 2018: Effect of vacuum freeze-drying on enhancing liquid permeability of moso bamboo. BioResources 13(2): 4159-4174.

17. Huang, Z.W., 2017: Effects of ultrasonic treatment on surface characteristics and bonding interface properties of bamboo laminated lumber. Pp 12-24, Nanjing Forestry University, Nanjing, China.

18. Rao, S., Yu, L.P., 2013: Effect of pretreatment on fluid permeability of moso bamboo. Forestry Machinery\&Woodworking Equipment 41(8): 26-29.

19. Du, C.G., Wei, J.G., Jin, C.D., 2016: Fire retardant treatment process of bamboo scrimber. Journal of Forestry Engineering 1(1): 55-54.

20. Yu, L.L., Cai, J., Lu, F., Qin, D.C., Fei, B.H., 2017: Combustibility of boron-containing fire retardant treated bamboo filament. Wood and Fiber Science 49(2): 125-133.

21. Jin, C.D., Li, J.P., Yao, Q.F., 2015: A novel electrically conductive material derived from in situ synthesis of silver nanoparticles on the surface of bamboo timer. Wood Research 60(4): 617-621.

22. Li, H., Yang, Z.B., Yang, F., Gu, Z.C., Yu, L.L., Ma X.X., Fei B.H., 2018: Influence of four coatings on the mold-resistance and combustion performance of decorative bamboo curtain. Wood and Fiber Science 50(4): 447-457.

23. Li, H., Chen, M.L., Lv, H.F., Fei, B.H., 2018: Effect of flame retardant treatments on the antimold and combustion properties of decorative bamboo filament. Journal of Central South University of Forestry \& Technology 38(7): 110-116.

24. Wang, Q.W., Li, J., Winandy, J.E., 2004: Chemical mechanism of fire retardance of boric acid on wood. Wood Science and Technology 38: 375-389

25. Zhang, Y.H., Cai, J.W., Li, Z. 2018: Research on improving mechanical and flame retardant properties of bamboo. Chemical Research 29(2): 197-201. 
Chunyan Li, Xinjie Zhou, Changhao Yang, Lili Yu*

Tianjin University Of Science And Technology

Faculty Of Light Industry Science And Engineering

Dagu Southroad io38, Hexi District, P. O. Box 546 300222 Tianjin

P. R. China

*Corresponding author: yulilucky@tust.edu.cn

\author{
HuI LI* \\ 1Hubei Academy Of Forestry \\ Wuhan 430075, Hubei \\ P. R. CHINA
}

2National Positioning Observation And Research Station

of Bamboo Forest Ecosystem In Mufu Mountain

Xianning 437ioo, Hubei

P. R. China

Benhua Fei*

International Center For Bamboo And Rattan

Futong Eastroad 8, Chaoyang District

IOOIO2 BEIJING

P. R. China 
WOOD RESEARCH 